\title{
Robust Coordinated Formation for Multiple Surface Vessels Based on Backstepping Sliding Mode Control
}

\author{
Mingyu Fu, ${ }^{1}$ Jianfang Jiao, ${ }^{1}$ and Shen Yin ${ }^{2}$ \\ ${ }^{1}$ College of Automation, Harbin Engineering University, Harbin, Heilongjiang 150001, China \\ ${ }^{2}$ University of Duisburg-Essen, 47057 Duisburg, Germany \\ Correspondence should be addressed to Jianfang Jiao; jiaojianfangheu@163.com
}

Received 13 June 2013; Accepted 25 July 2013

Academic Editor: Lixian Zhang

Copyright (c) 2013 Mingyu Fu et al. This is an open access article distributed under the Creative Commons Attribution License, which permits unrestricted use, distribution, and reproduction in any medium, provided the original work is properly cited.

\begin{abstract}
We investigate the problem of coordinated formation control for multiple surface vessels in the presence of unknown external disturbances. In order to realize the leaderless coordinated formation and achieve the robustness against unknown external disturbances, a new robust coordinated formation control algorithm based on backstepping sliding mode control is proposed. The proposed coordinated control algorithm is achieved by defining a new switched function using the combination of position tracking error and cross-coupling error. Particularly, the cross-coupling error is defined using velocity tracking error and velocity synchronization error so as to be applicable for sliding mode controller design. Furthermore, the adaptive control law is proposed to estimate unknown disturbances for each vessel. The globally asymptotically stability is proved using the Lyapunov direct method. Finally, the effectiveness of the proposed coordinated formation control algorithm is demonstrated by corresponding simulations.
\end{abstract}

\section{Introduction}

Recently, coordination and consensus of multiagent systems have received much attention, and the network-induced constraints are discussed [1]. Many studies on coordination control issues have been widely reported in the existing literature due to the widespread applications, such as multiple mobile robots, spacecraft formation, networked sensors, vehicles coordination [2]. Particulary, the applications of formation control at sea are increasing, which can be found within both civil and military operations. For example, a group of surface vessels travel in formation structures to perform the seabed mapping operations, the collective range of sensor equipment can be maximized, ensuring that larger areas can be covered in shorter time compared with a single vessel. And another example is underway replenishment of vessels which is typically performed by one or more supply vessels lining up at the side of the receiving vessel, after which all vessels strive to maintain equal and constant forward speed and bearing while supplies are being transferred across messenger lines [3]. The complicated operations often cannot be carried out through only one vessel.
In a word, multiple vessels work together to improve performance and reduce fatigue and difficulty for the people involved. What is more, compared with a single vessel, multiple vessels perform the complicated tasks with less time and cost in practical maritime. So this paper will focus on the coordinated formation of multiple surface vessels; And the formation control in this paper aims at the surface vessels, then the robustness to environmental disturbances is highly important when considering the marine and offshore applications. So the study on the robust coordination control algorithm for multiple surface vessels is significative.

With respect to the coordination control issues of multiple surface vessels, abundant studies have been widely reported in the existing literature. There are several typical approaches used to design the coordination formation controller. For example, Lagrangian formation method [4], nullspace-based behavioral control [5], nonlinear model predictive control [6], and graph theory [7]. Meanwhile the problem of coordinated path following for multiple vessels also has been discussed in the following references [8-10]. In recent years, the formation control of multiple vessels is researched using the passivity-based control method included in the 
synchronization control approach [11-13]. A common trait in the aforementioned results is that the coordinated tracking controller is designed by assuming that motions of vessels are disturbance-free. However, surface vessels often encounter external disturbances such as wind, wave, and current. The external environment disturbances are difficult to model well and truly because of the disturbances varying with weather conditions and depth of water area change. So the coordinated formation algorithm for multiple surface vessels should be robust to unknown disturbances. The adaptive control is helpful for solving this problem $[14,15]$.

This paper develops a coordinated formation control algorithm based on backstepping sliding mode control approach. The sliding mode control possesses the robustness to system uncertainty and external disturbance as a result of the definition of the switched function [16]. And the stabilization for the switched systems are discussed in $[17,18]$. For a single surface vessel, the sliding mode control method can achieve robustness to the environment disturbances $[19,20]$. Furthermore, the sliding mode control is also used to design the coordinated formation controller as in reference [21, 22]. And the backstepping method can determine the appropriate Lyapunov function systematically and simply, which makes the distributed robust and adaptive redesign implementable. The integration of backstepping and sliding mode control will centralize both the advantages of the two control schemes [23]. Backstepping sliding mode control is used to solve the coordinated formation problem for multi-agent systems in $[24,25]$. The aforementioned studies on sliding mode formation control are all based on the leader-follower strategy. However, the leaderless strategy is applicable widely because of most practical task without emphasizing which vessel is important [26]. The cross-coupling synchronization approach is propitious to the leaderless formation controller design. In addition, the synchronization control approach has a simple control structure and convenient implementation capability compared to existing formation control approach. The cross-coupling synchronization error can be convenient for defining switched function in the sliding mode controller design [27]. However, the cross-coupling error of [27] is composed of position tracking error and integral of synchronization error, which cannot be applicable to design sliding mode controller. This motivates that the cross-coupling error is redefined to be applicable to the whole coordinated controller design.

In this paper, a robust coordinated formation controller based on backstepping sliding mode control is proposed for multiple surface vessels. And a new switched function is defined using a new cross-coupling error to achieve leaderless coordination between these vessels. And the cross-coupling error is defined using the velocity error and the synchronization velocity error. This will be applicable to the sliding mode controller design. Furthermore, with respect to the unknown disturbances, the adaptive control law is proposed to improve the robust coordinated formation control algorithm. The rest of this paper is organized as follows. Section 2 introduces the basic notations for the graph theory and establishes the vessel model. Section 3 describes the proposed coordination control algorithm in detail. Section 4 describes robust coordinated tracking controller. The simulation of the proposed coordination algorithm for five vessels is carried out, and the validity of the proposed coordinated control algorithm is demonstrated in Section 5. At last, we draw conclusions in Section 6.

\section{Preliminaries}

2.1. Notations. In this section, several basic concepts about the directed connected graph are given. A directed graph G consists of the pair $(\nu, \varepsilon) . v$ is a set of vertices; $\varepsilon \in \nu^{2}$ is a set of edges. $(i, j) \in \varepsilon$ if information flows from vertex $j$ to vertex $i$. If any two distinct vertices of a directed graph can be connected through a directed path, then the directed graph is called strongly connected. The adjacent matrix of the directed graph is denoted as $\mathbf{A} \in \mathrm{R}^{n \times n}$, which is defined as diagonal entries 0 and off-diagonal entries $a_{i j}=1$ if $(i, j) \in \varepsilon$ and 0 otherwise. And the degree matrix $\mathbf{D} \in \mathrm{R}^{n \times n}$ is defined with off-diagonal entries 0 and diagonal entries $d_{i i}=\sum_{j \neq i} a_{i j}$. Then the Laplacian matrix is obtained as $\mathbf{L}=\mathbf{D}-\mathbf{A} \in \mathrm{R}^{n \times n}$. That is $\mathbf{L}=\left[l_{i j}\right] \in \mathrm{R}^{n \times n}$, and $l_{i i}=\sum_{j \neq i} a_{i j}, l_{i j}=-a_{i j}$.

In this paper, the communication topology between these vessels is described by a strongly connected graph. Each vertex in the graph represents a vessel in the group. The edges represent information exchange links by available communication.

Define the Kronecker product of two matrices $\mathbf{A} \in \mathrm{R}^{m \times n}$ and $\mathbf{B} \in \mathrm{R}^{p \times q}$ as

$$
\mathbf{A} \otimes \mathbf{B}=\left[\begin{array}{ccc}
a_{11} \mathbf{B} & \cdots & a_{1 n} \mathbf{B} \\
\vdots & \ddots & \vdots \\
a_{m 1} \mathbf{B} & \cdots & a_{m n} \mathbf{B}
\end{array}\right] .
$$

Theorem 1 (see [28]). Let $x=0$ be an equilibrium point for $\dot{x}=f(t, x)$, and let $D \subset \mathrm{R}^{n}$ be a domain containing $x=0$. Let $V:[0, \infty) \times D \rightarrow \mathrm{R}$ be a continuously differentiable function such that

$$
\begin{aligned}
& W_{1}(x) \leq V(t, x) \leq W_{2}(x), \\
& \frac{\partial V}{\partial t}+\frac{\partial V}{\partial x} f(t, x) \leq W_{3}(x)
\end{aligned}
$$

for all $t \geq 0$ and for all $x \subset D$, where $W_{1}(x), W_{2}(x)$, and $W_{3}(x)$ are continuous positive definite functions on $D$. Then, $x=0$ is uniformly asymptotically stable. If $D=\mathrm{R}^{n}$ and $W_{1}(x)$ is radially unbounded, then $x=0$ is globally uniformly asymptotically stable.

Lemma 2 (Barbalat's lemma [28]). Let $\phi: \mathrm{R} \rightarrow \mathrm{R}$ be a uniformly continuous function on $[0, \infty)$. Suppose that $\lim _{t \rightarrow \infty} \int_{0}^{t} \phi(\tau) d \tau$ exists and is finite. Then, $\phi(t) \rightarrow 0$ as $t \rightarrow \infty$.

2.2. Mathematical Vessel Model. The vessel model can be divided into two parts: the kinematics and nonlinear dynamics. Generally, only the motion in the horizontal plane is considered for the surface vessel. The elements corresponding to heave, roll, and pitch are neglected. The dynamic model for 
the $i$ th surface vessel can be represented by the following 3 degrees of freedom (DOF) [29]:

$$
\begin{gathered}
\dot{\eta}_{i}=\mathbf{R}_{i}\left(\psi_{i}\right) \mathbf{v}_{i} \\
\mathbf{M}_{i} \dot{\mathbf{v}}_{i}+\mathbf{C}_{i}\left(\mathbf{v}_{i}\right) \mathbf{v}_{i}+\mathbf{D}_{i}\left(\mathbf{v}_{i}\right) \mathbf{v}_{i}=\tau_{i}+\tau_{d i}
\end{gathered}
$$

where $\eta_{i}=\left[n_{i}, e_{i}, \psi_{i}\right]^{T}$ denotes the north position, east position, and orientation which are decomposed in the earthfixed reference frame, and $\mathbf{v}_{i}=\left[u_{i}, v_{i}, r_{i}\right]^{T}$ denotes the linear surge velocity, sway velocity and angular velocity, which are decomposed in the body-fixed reference frame. $\mathbf{R}_{i}\left(\psi_{i}\right)$ is the transformations matrix from the body-fixed reference frame to the earth-fixed reference frame, the form of which is as follows:

$$
\mathbf{R}_{i}\left(\psi_{i}\right)=\left[\begin{array}{ccc}
\cos \left(\psi_{i}\right) & -\sin \left(\psi_{i}\right) & 0 \\
\sin \left(\psi_{i}\right) & \cos \left(\psi_{i}\right) & 0 \\
0 & 0 & 1
\end{array}\right] .
$$

Then we can know that the transformations matrix satisfies the following properties as $\mathbf{R}_{i}^{-1}\left(\psi_{i}\right)=\mathbf{R}_{i}^{T}\left(\psi_{i}\right)$, for all $\psi_{i}$. $\mathbf{M}_{i}$ denotes the system inertia mass matrix including added mass which is positive definite. $\mathbf{C}_{i}\left(\mathbf{v}_{i}\right)$ and $\mathbf{D}_{i}\left(\mathbf{v}_{i}\right)$ denote the Corioliscentripetal matrix and damping matrix, respectively. The detailed representation of the previous three-system matrix can be found in [29]. $\tau_{i}=\left[\begin{array}{lll}\tau_{u i} & \tau_{v i} & \tau_{r i}\end{array}\right]^{T}$ is the vector of forces and torques input from the thruster system. $\tau_{d i}$ is the vector of external environment forces and torques input which are generating by wind, wave and current.

In order to design the backstepping sliding mode controller, we transform the vessel model as following. Equation (3) can be transformed as

$$
\mathbf{v}_{i}=\mathbf{R}_{i}^{-1}\left(\psi_{i}\right) \dot{\eta}_{i}
$$

By differentiating $\mathbf{R}_{i}\left(\psi_{i}\right)$, we have $\dot{\mathbf{R}}_{i}\left(\psi_{i}\right)=\mathbf{R}_{i}\left(\psi_{i}\right) S$, where

$$
S=\left[\begin{array}{ccc}
0 & -r_{i} & 0 \\
r_{i} & 0 & 0 \\
0 & 0 & 0
\end{array}\right]=-S^{T},
$$

where $r_{i}$ is the angular velocity in the body-fixed reference frame. Take the derivative of (6), we can obtain that:

$$
\begin{aligned}
\dot{\mathbf{v}}_{i} & =\dot{\mathbf{R}}_{i}^{-1}\left(\psi_{i}\right) \dot{\eta}_{i}+\mathbf{R}_{i}^{-1}\left(\psi_{i}\right) \ddot{\eta}_{i} \\
& =-\mathbf{R}_{i}^{-1}\left(\psi_{i}\right) \dot{\mathbf{R}}_{i}\left(\psi_{i}\right) \mathbf{R}_{i}^{-1}\left(\psi_{i}\right) \dot{\eta}_{i}+\mathbf{R}_{i}^{-1}\left(\psi_{i}\right) \ddot{\eta}_{i} .
\end{aligned}
$$

Taking (6) and (8) into the vessel dynamic model (4) can yield

$$
\begin{aligned}
& \mathbf{M}_{i} \mathbf{R}_{i}^{-1}\left(\psi_{i}\right) \ddot{\eta}_{i}-\mathbf{M}_{i} \mathbf{R}_{i}^{-1}\left(\psi_{i}\right) \dot{\mathbf{R}}_{i}\left(\psi_{i}\right) \mathbf{R}_{i}^{-1}\left(\psi_{i}\right) \dot{\eta}_{i} \\
& \quad+\mathbf{C}_{i}\left(\mathbf{v}_{i}\right) \mathbf{R}_{i}^{-1}\left(\psi_{i}\right) \dot{\eta}_{i}+\mathbf{D}_{i}\left(\mathbf{v}_{i}\right) \mathbf{R}_{i}^{-1}\left(\psi_{i}\right) \dot{\eta}_{i}=\tau_{i}+\tau_{d i} .
\end{aligned}
$$

The above equation can be written as

$$
\mathbf{M}_{n i}\left(\eta_{i}\right) \ddot{\eta}_{i}+\mathbf{C}_{n i}\left(\eta_{i}, \dot{\eta}_{i}\right) \dot{\eta}_{i}+\mathbf{D}_{n i}\left(\eta_{i}, \dot{\eta}_{i}\right) \dot{\eta}_{i}=\tau_{i}+\tau_{d i},
$$

where

$$
\begin{gathered}
\mathbf{M}_{n i}\left(\eta_{i}\right)=\mathbf{M}_{i} \mathbf{R}_{i}^{-1}\left(\psi_{i}\right) ; \\
\mathbf{C}_{n i}\left(\eta_{i}, \dot{\eta}_{i}\right)=\left[\mathbf{C}_{i}\left(\mathbf{v}_{i}\right)-\mathbf{M}_{i} \mathbf{R}_{i}^{-1}\left(\psi_{i}\right) \dot{\mathbf{R}}_{i}\left(\psi_{i}\right)\right] \mathbf{R}_{i}^{-1}\left(\psi_{i}\right) ; \\
\mathbf{D}_{n i}\left(\eta_{i}, \dot{\eta}_{i}\right)=\mathbf{D}_{i}\left(\mathbf{v}_{i}\right) \mathbf{R}_{i}^{-1}\left(\psi_{i}\right) .
\end{gathered}
$$

\section{Coordinated Formation Controller Design}

In this section, we will design the coordinated trajectory tracking controller for multiple surface vessels based on backstepping sliding mode control method. And we assume that all the vessels are disturbance-free. The detailed procedure of controller design is as follows.

3.1. Formation Setup. This paper considers a fleet of $n$ vessels to perform the desired coordination formation task. And each vessel in the formation is identified by the index set $I=$ $[1,2, \ldots, n]$. As in [12], the desired formation is established by defining the formation reference vector for each vessel, which is denoted as $\mathbf{1}_{i}=\left[x_{0 i}, y_{0 i}, \psi_{0 i}\right]^{T}$, where $x_{0 i}, y_{0 i}, \psi_{0 i}$ are constant, respectively, and $-\pi \leq \psi_{0 i} \leq \pi$. Then the formation reference point for each vessel is given by: $\mathbf{x}_{i}=\eta_{i}+\mathbf{R}_{i}\left(\psi_{i}-\right.$ $\left.\psi_{0 i}\right) \mathbf{l}_{i}$. If we assume the desired trajectory of the formation point is denoted as $\eta_{d}$, where $\eta_{d}=\left[n_{d}(t), e_{d}(t), \psi_{d}(t)\right]^{T}$, $n_{d}(t), e_{d}(t)$ are sufficiently smooth functions, and $\psi_{d}(t)=$ $\arctan \left(\dot{e}_{d}(t) / \dot{n}_{d}(t)\right)$. That means that the vessel direction is chosen as the tangential vector of the respective desired trajectory. We can know that the coordinated formation is achieved if all the formation reference points of the group of vessels are synchronized; that is $\mathbf{x}_{1}=\cdots=\mathbf{x}_{i}=\cdots=\mathbf{x}_{n}=\eta_{d}$.

3.2. Formation Controller Design. The proposed coordinated formation controller for multiple surface vessels is designed using the backstepping sliding mode control approach, and a new switched function is defined to accomplish the sliding mode controller.

Define the position tracking error of the formation reference point for each vessel as

$$
\mathbf{z}_{1 i}=\mathbf{x}_{i}-\eta_{d}=\eta_{i}+\mathbf{R}_{i}\left(\psi_{i}-\psi_{0 i}\right) \mathbf{l}_{i}-\eta_{d} .
$$

If we define the new variable $\mathbf{f}_{i}$ as $\mathbf{f}_{i}=\mathbf{R}_{i}\left(\psi_{i}-\psi_{0 i}\right) \mathbf{l}_{i}$, take the derivative of $\mathbf{z}_{1 i}$, we can obtain that

$$
\dot{\mathbf{z}}_{1 i}=\dot{\eta}_{i}+\dot{\mathbf{f}}_{i}-\dot{\eta}_{d}
$$

Define the following stabilizing function for each vessel as

$$
\alpha_{i}=\lambda_{i} \mathbf{z}_{1 i}
$$

where $\lambda_{i} \in \mathrm{R}^{3 \times 3}$ is a diagonal positive definite matrix.

Define the velocity error as $\mathbf{z}_{2 i}$; the form of which is

$$
\mathbf{z}_{2 i}=\dot{\mathbf{z}}_{1 i}+\alpha_{i}=\dot{\mathbf{z}}_{1 i}+\lambda_{i} \mathbf{z}_{1 i} .
$$

Then we can obtain

$$
\mathbf{z}_{2 i}=\dot{\eta}_{i}+\dot{\mathbf{f}}_{i}-\dot{\eta}_{d}+\lambda_{i} \mathbf{z}_{1 i}
$$

With the form of vessel model, then we have

$$
\begin{aligned}
\mathbf{M}_{n i}\left(\eta_{i}\right) \dot{\mathbf{z}}_{2 i}= & \mathbf{M}_{n i}\left(\eta_{i}\right)\left(\ddot{\eta}_{i}+\ddot{\mathbf{f}}_{i}-\ddot{\eta}_{d}+\lambda_{i} \mathbf{z}_{1 i}\right) \\
= & \tau_{i}+\tau_{d i}-\mathbf{C}_{n i}\left(\eta_{i}, \dot{\eta}_{i}\right) \dot{\eta}_{i}-\mathbf{D}_{n i}\left(\eta_{i}, \dot{\eta}_{i}\right) \dot{\eta}_{i} \\
& +\mathbf{M}_{n i}\left(\eta_{i}\right) \ddot{\mathbf{f}}_{i}-\mathbf{M}_{n i}\left(\eta_{i}\right) \ddot{\eta}_{d}+\mathbf{M}_{n i}\left(\eta_{i}\right) \lambda_{i} \mathbf{z}_{1 i} .
\end{aligned}
$$


In light of (16), we can obtain that

$$
\dot{\eta}_{i}=\dot{\eta}_{d}-\dot{\mathbf{f}}_{i}+\mathbf{z}_{2 i}-\lambda_{i} \mathbf{z}_{1 i}
$$

Then (17) can be calculated as follows

$$
\begin{aligned}
\mathbf{M}_{n i}\left(\eta_{i}\right) \dot{\mathbf{z}}_{2 i}= & \tau_{i}+\tau_{d i}-\left(\mathbf{C}_{n}\left(\eta_{i}, \dot{\eta}_{i}\right)+\mathbf{D}_{n}\left(\eta_{i}, \dot{\eta}_{i}\right)\right)\left(\dot{\eta}_{d}-\dot{\mathbf{f}}_{i}\right) \\
& -\left(\mathbf{C}_{n i}\left(\eta_{i}, \dot{\eta}_{i}\right)+\mathbf{D}_{n i}\left(\eta_{i}, \dot{\eta}_{i}\right)\right)\left(\mathbf{z}_{2 i}-\lambda_{i} \mathbf{z}_{1 i}\right) \\
& -\mathbf{M}_{n i}\left(\eta_{i}\right)\left(\ddot{\eta}_{d}-\ddot{\mathbf{f}}\right)+\mathbf{M}_{n i}\left(\eta_{i}\right) \lambda_{i} \dot{\mathbf{z}}_{1 i} .
\end{aligned}
$$

For representing conveniently, we define $\mathbf{z}_{1}=\left[\begin{array}{lll}z_{11}^{T} & z_{12}^{T} & \cdots\end{array}\right.$ $\left.z_{1 n}^{T}\right]^{T} ; \mathbf{z}_{2}=\left[\begin{array}{llll}z_{21}^{T} & z_{22}^{T} & \cdots & z_{2 n}^{T}\end{array}\right]^{T} ; \eta=\left[\begin{array}{llll}\eta_{1}^{T} & \eta_{2}^{T} & \cdots & \eta_{n}^{T}\end{array}\right]^{T} ; \mathbf{f}=$ $\left[\begin{array}{llll}\mathbf{f}_{1}^{T} & \mathbf{f}_{2}^{T} & \cdots & \mathbf{f}_{n}^{T}\end{array}\right]^{T} ; \tau=\left[\begin{array}{llll}\tau_{1}^{T} & \tau_{2}^{T} & \cdots & \tau_{n}^{T}\end{array}\right]^{T} ; \tau_{d}=\left[\begin{array}{lll}\tau_{d 1}^{T} & \tau_{d 2}^{T} & \cdots\end{array}\right.$ $\left.\tau_{d n}^{T}\right]^{T} ; \eta_{D}=\left[\begin{array}{llll}\eta_{d}^{T} & \eta_{d}^{T} & \cdots & \eta_{d}^{T}\end{array}\right]^{T} ;$

$$
\begin{aligned}
& \lambda=\operatorname{diag}\left(\begin{array}{llll}
\lambda_{1} & \lambda_{2} & \cdots & \lambda_{n}
\end{array}\right) \\
& \mathbf{M}_{n}(\eta)=\operatorname{diag}\left(\mathbf{M}_{n 1}\left(\eta_{1}\right) \cdots \mathbf{M}_{n n}\left(\eta_{n}\right)\right) \text {, } \\
& \mathbf{C}_{n}(\eta, \dot{\eta})=\operatorname{diag}\left(\mathbf{C}_{n 1}\left(\eta_{1}, \dot{\eta}_{1}\right) \cdots \mathbf{C}_{n n}\left(\eta_{n}, \dot{\eta}_{n}\right)\right) \text {, } \\
& \mathbf{D}_{n}(\eta, \dot{\eta})=\operatorname{diag}\left(\mathbf{D}_{n 1}\left(\eta_{1}, \dot{\eta}_{1}\right) \cdots \mathbf{D}_{n n}\left(\eta_{n}, \dot{\eta}_{n}\right)\right) \text {. }
\end{aligned}
$$

So the error dynamics of multiple surface vessels can be written in terms of matrix and vector:

$$
\begin{gathered}
\dot{\mathbf{z}}_{1}=\mathbf{z}_{2}-\lambda \mathbf{z}_{1}, \\
\dot{\mathbf{z}}_{2}=\mathbf{M}_{n}^{-1}(\eta)\left[\tau+\tau_{d}-\mathbf{M}_{n}(\eta)\left(\ddot{\eta}_{D}-\ddot{\mathbf{f}}\right)\right. \\
-\left(\mathbf{C}_{n}(\eta, \dot{\eta})+\mathbf{D}_{n}(\eta, \dot{\eta})\right)\left(\dot{\eta}_{D}-\dot{\mathbf{f}}\right) \\
-\left(\mathbf{C}_{n}(\eta, \dot{\eta})+\mathbf{D}_{n}(\eta, \dot{\eta})\right)\left(\mathbf{z}_{2}-\lambda \mathbf{z}_{1}\right) \\
\left.+\mathbf{M}_{n}(\eta) \lambda \dot{\mathbf{z}}_{1}\right] .
\end{gathered}
$$

Define the synchronization velocity error vector for these vessels as

$$
\varepsilon=\left(\mathbf{L} \otimes \mathbf{I}_{3}\right) \mathbf{z}_{2}
$$

where $\mathbf{L} \in \mathrm{R}^{n \times n}$ is the Laplacian matrix of the communication topology graph for these vessels.

Define the cross-coupling error using the velocity error and the synchronization velocity error; the form of which is

$$
\mathbf{e}=\mathbf{z}_{2}+\gamma \varepsilon=\mathbf{z}_{2}+\gamma\left(\mathbf{L} \otimes \mathbf{I}_{3}\right) \mathbf{z}_{2}=\left(\mathbf{I}_{3 n}+\gamma\left(\mathbf{L} \otimes \mathbf{I}_{3}\right)\right) \mathbf{z}_{2},
$$

where $\gamma \in \mathrm{R}^{3 n \times 3 n}$ is a diagonal positive definite matrix.

The switched function is defined using the position tracking error and the cross-coupling error. The detailed form is as follows

$$
\begin{aligned}
\mathbf{s} & =\mathbf{k z}_{1}+\mathbf{e} \\
& =\mathbf{k z}_{1}+\left(\mathbf{I}_{3 n}+\gamma\left(\mathbf{L} \otimes \mathbf{I}_{3}\right)\right) \mathbf{z}_{2},
\end{aligned}
$$

where $\mathbf{k} \in \mathrm{R}^{3 n \times 3 n}$ is a diagonal positive definite matrix.
If we assume these vessels are disturbance-free; that is $\boldsymbol{\tau}_{d}=0$, then the control input can be chosen as follows

$$
\tau=\tau_{\mathrm{eq}}+\tau_{\mathrm{sw}}
$$

where $\tau_{\mathrm{eq}}$ is the equivalent control input, and $\tau_{\mathrm{sw}}$ is the switch control part of the backstepping sliding mode control input. The detailed expressions are as follows:

$$
\begin{aligned}
\tau_{\mathrm{eq}}= & \mathbf{M}_{n}(\eta)\left(\ddot{\eta}_{D}-\ddot{\mathbf{f}}\right)-\mathbf{M}_{n}(\eta) \lambda \dot{\mathbf{z}}_{1} \\
& +\left(\mathbf{C}_{n}(\eta, \dot{\eta})+\mathbf{D}_{n}(\eta, \dot{\eta})\right)\left(\dot{\eta}_{D}-\dot{\mathbf{f}}\right) \\
& +\left(\mathbf{C}_{n}(\eta, \dot{\eta})+\mathbf{D}_{n}(\eta, \dot{\eta})\right)\left(\mathbf{z}_{2}-\lambda \mathbf{z}_{1}\right) \\
& -\mathbf{M}_{n}(\eta)\left(\mathbf{I}_{3 n}+\gamma\left(\mathbf{L} \otimes \mathrm{I}_{3}\right)\right)^{-1} \mathbf{k}\left(\mathbf{z}_{2}-\lambda \mathbf{z}_{1}\right), \\
\tau_{\mathrm{sw}}= & -\mathbf{M}_{n}\left(\mathbf{I}_{3 n}+\gamma\left(\mathbf{L} \otimes \mathrm{I}_{3}\right)\right)^{-1} \mathbf{P}(\mathbf{s}+\beta \operatorname{sign}(\mathbf{s})),
\end{aligned}
$$

where $\mathbf{P} \in \mathrm{R}^{3 n \times 3 n}, \beta \in \mathrm{R}^{3 n \times 3 n}$ are a diagonal positive definite matrix, respectively. And $\operatorname{sign}(\cdot)$ is sign function. And $\operatorname{sign}(\mathbf{s})=\left[\begin{array}{llll}\operatorname{sign}\left(\mathbf{s}_{1}^{T}\right) & \operatorname{sign}\left(\mathbf{s}_{2}^{T}\right) & \cdots & \operatorname{sign}\left(\mathbf{s}_{n}^{T}\right)\end{array}\right]^{T}$.

Remark 3. The control input vector for each vessel which is disturbance-free can be written as

$$
\tau_{i}=\tau_{\mathrm{eq}}^{i}+\tau_{\mathrm{sw}}^{i}
$$

Define

$$
\begin{aligned}
\mathbf{Y}=\left(\mathbf{I}_{3 n}+\gamma\left(\mathbf{L} \otimes \mathbf{I}_{3}\right)\right)^{-1}=\left\{Y_{i j} \in \mathrm{R}^{3 \times 3}\right\} \in \mathrm{R}^{3 n \times 3 n}, \\
\gamma=\operatorname{diag}\left(\begin{array}{llll}
\gamma_{1} & \gamma_{2} & \cdots & \gamma_{n}
\end{array}\right), \\
\mathbf{k}=\operatorname{diag}\left(\begin{array}{llll}
\mathbf{k}_{1} & \mathbf{k}_{2} & \cdots & \mathbf{k}_{n}
\end{array}\right), \\
\mathbf{P}=\operatorname{diag}\left(\begin{array}{lllll}
\mathbf{P}_{1} & \mathbf{P}_{2} & \cdots & \mathbf{P}_{n}
\end{array}\right) \\
\beta=\operatorname{diag}\left(\begin{array}{lllll}
\beta_{1} & \beta_{2} & \cdots & \beta_{n}
\end{array}\right) .
\end{aligned}
$$

The detailed expressions of the control input for each vessel are as follows:

$$
\begin{aligned}
\tau_{\mathrm{eq}}^{i}= & \mathbf{M}_{n i}\left(\eta_{i}\right)\left(\ddot{\eta}_{d}-\ddot{\mathbf{f}}_{i}\right)-\mathbf{M}_{n i}\left(\eta_{i}\right) \lambda \dot{\mathbf{z}}_{1 i} \\
& +\left(\mathbf{C}_{n i}\left(\eta_{i}, \dot{\eta}_{i}\right)+\mathbf{D}_{n i}\left(\eta_{i}, \dot{\eta}_{i}\right)\right)\left(\dot{\eta}_{d}-\dot{\mathbf{f}}_{i}\right) \\
& +\left(\mathbf{C}_{n i}\left(\eta_{i}, \dot{\eta}_{i}\right)+\mathbf{D}_{n i}\left(\eta_{i}, \dot{\eta}_{i}\right)\right)\left(\mathbf{z}_{2 \mathbf{i}}-\lambda_{i} \mathbf{z}_{1 i}\right) \\
& -\mathbf{M}_{n i}\left(\eta_{i}\right) \sum_{j=1}^{n}\left[Y_{i j}\left(k_{j}\left(\mathbf{z}_{2 j}-\lambda_{j} \mathbf{z}_{1 j}\right)\right)\right] \\
\tau_{\mathrm{sw}}^{i}= & -\mathbf{M}_{n i}\left(\eta_{i}\right) \sum_{j=1}^{n}\left[Y_{i j} \mathbf{P}_{j}\left(\mathbf{s}_{j}+\beta_{j} \operatorname{sign}\left(\mathbf{s}_{j}\right)\right)\right],
\end{aligned}
$$

where the switched function is defined as

$$
\mathbf{s}_{j}=\mathbf{k}_{j} \mathbf{z}_{1 j}+\mathbf{z}_{2 j}+\gamma_{j} \sum_{i=1}^{n} a_{j i}\left(\mathbf{z}_{2 i}-\mathbf{z}_{2 j}\right),
$$

where $\mathbf{A}=\left\{a_{j i}\right\} \in \mathrm{R}^{n \times n}$ is the adjacency matrix for the communication topology graph. $a_{i i}=0$ and $a_{j i}=1$ if information flow from vessel $i$ to vessel $j$ and 0 otherwise, for all $j \neq i$. 
Theorem 4. Consider a group of $n$ vessels to perform the coordination formation task; the vessel model is described as (3) and (4), and we assume that these vessels are disturbance-free. With the distributed coordinated formation control law (26) and (27), the following conditions are satisfied;

(i) all the matrices $\lambda, \mathbf{P}$, and $\mathbf{k}$ are diagonal positive definite;

(ii) $\left(\lambda \boldsymbol{\Gamma}^{T}+\mathbf{k}\right) \mathbf{P} \boldsymbol{\Gamma}-(1 / 4) \mathbf{I}_{3 n}>0$, where $\boldsymbol{\Gamma}=\mathbf{I}_{3 n}+\gamma\left(\mathbf{L} \otimes \mathbf{I}_{3}\right)$;

(iii) the matrix $\gamma$ is diagonal positive definite and small enough.

Then the position tracking error, the velocity error and their synchronization error, approach zero asymptoticallyl; that is, these vessels can realize the coordination formation asymptotically.

Proof. Define the first Lyapunov function as

$$
V_{1}=\frac{1}{2} \mathbf{z}_{1}^{T} \mathbf{z}_{1}
$$

Differentiating $V_{1}$ with respect to time, then we have

$$
\dot{V}_{1}=-\mathbf{z}_{1}^{T} \lambda \mathbf{z}_{1}+\mathbf{z}_{1}^{T} \mathbf{z}_{2} .
$$

Define the second Lyapunov function as

$$
V_{2}=V_{1}+\frac{1}{2} \mathbf{s}^{T} \mathbf{s}
$$

Take the time derivative of the above equation as

$$
\begin{aligned}
\dot{V}_{2}= & \dot{V}_{1}+\mathbf{s}^{T} \dot{\mathbf{s}} \\
= & \dot{V}_{1}+\mathbf{s}^{T} \mathbf{k} \dot{\mathbf{z}}_{1}+\mathbf{s}^{T}\left(\mathbf{I}_{3 n}+\gamma\left(\mathbf{L} \otimes \mathrm{I}_{3}\right)\right) \dot{\mathbf{z}}_{2} \\
= & \mathbf{z}_{1}^{T} \mathbf{z}_{2}-\mathbf{z}_{1}^{T} \lambda \mathbf{z}_{1}+\mathbf{s}^{T} \mathbf{k}\left(\mathbf{z}_{2}-\lambda \mathbf{z}_{1}\right) \\
& +\mathbf{s}^{T}\left(\mathbf{I}_{3 n}+\gamma\left(\mathbf{L} \otimes \mathbf{I}_{3}\right)\right) \mathbf{M}_{n}^{-1}(\eta) \\
& \times\left[\tau-\mathbf{M}_{n}(\eta) \ddot{\eta}_{D}+\mathbf{M}_{n}(\eta) \dot{\mathbf{f}}+\mathbf{M}_{n}(\eta) \lambda \dot{\mathbf{z}}_{1}\right. \\
& \quad-\left(\mathbf{C}_{n}(\eta, \dot{\eta})+\mathbf{D}_{n}(\eta, \dot{\eta})\right)\left(\dot{\eta}_{D}-\dot{\mathbf{f}}\right) \\
& \left.\quad-\left(\mathbf{C}_{n}(\eta, \dot{\eta})+\mathbf{D}_{n}(\eta, \dot{\eta})\right)\left(\mathbf{z}_{2}-\lambda \mathbf{z}_{1}\right)\right] .
\end{aligned}
$$

With the control input (26) and (27), we can obtain that

$$
\begin{aligned}
\dot{V}_{2} & =-\mathbf{z}_{1}^{T} \lambda \mathbf{z}_{1}+\mathbf{z}_{1}^{T} \mathbf{z}_{2}-\mathbf{s}^{T} \mathbf{P}(\mathbf{s}+\beta \operatorname{sign}(\mathbf{s})) \\
& =-\mathbf{z}_{1}^{T} \lambda \mathbf{z}_{1}+\mathbf{z}_{1}^{T} \mathbf{z}_{2}-\mathbf{s}^{T} \mathbf{P} \mathbf{s}-\mathbf{s}^{T} \mathbf{P} \beta \operatorname{sign}(\mathbf{s}) \\
& =-\mathbf{z}_{1}^{T} \lambda \mathbf{z}_{1}+\mathbf{z}_{1}^{T} \mathbf{z}_{2}-\mathbf{s}^{T} \mathbf{P} \mathbf{s}-\sum_{r=1}^{3 n} p_{r} \beta_{r}\left|s_{r}\right|
\end{aligned}
$$

where $\left|s_{r}\right|$ denotes the absolute value of the variable of $s_{r}$.

If we define $\boldsymbol{\Gamma}=\mathbf{I}_{3 n}+\gamma\left(\mathbf{L} \otimes \mathbf{I}_{3}\right)$, then we choose matrix $\mathbf{Q}$ as

$$
\mathbf{Q}=\left[\begin{array}{cc}
\lambda+\mathbf{k}^{T} \mathbf{P} \mathbf{k} & \mathbf{k}^{T} \mathbf{P} \boldsymbol{\Gamma}-\frac{1}{2} \mathbf{I}_{3 n} \\
\boldsymbol{\Gamma}^{T} \mathbf{P}^{T} \mathbf{k}-\frac{1}{2} \mathbf{I}_{3 n} & \boldsymbol{\Gamma}^{T} \mathbf{P} \boldsymbol{\Gamma}
\end{array}\right]
$$

If we define $\mathbf{z}=\left[z_{1}^{T}, z_{2}^{T}\right]^{T}$, then we can obtain that

$$
\begin{aligned}
\mathbf{z}^{T} \mathbf{Q z}= & {\left[\begin{array}{ll}
\mathbf{z}_{1}^{T} & \mathbf{z}_{2}^{T}
\end{array}\right]\left[\begin{array}{cc}
\lambda+\mathbf{k}^{T} \mathbf{P} \mathbf{k} & \mathbf{k}^{T} \mathbf{P} \boldsymbol{\Gamma}-\frac{1}{2} \mathbf{I}_{3 n} \\
\boldsymbol{\Gamma}^{T} \mathbf{P k}-\frac{1}{2} \mathbf{I}_{3 n} & \boldsymbol{\Gamma}^{T} \mathbf{P} \boldsymbol{\Gamma}
\end{array}\right]\left[\begin{array}{l}
\mathbf{z}_{1} \\
\mathbf{z}_{2}
\end{array}\right] } \\
= & {\left[\left(\mathbf{z}_{1}^{T} \lambda+\mathbf{z}_{1}^{T} \mathbf{k}^{T} \mathbf{P} \mathbf{k}+\mathbf{z}_{2}^{T} \boldsymbol{\Gamma}^{T} \mathbf{P} \mathbf{k}-\frac{1}{2} \mathbf{z}_{2}^{T} \mathbf{I}_{3 n}\right)\right.} \\
& \left.\times\left(\mathbf{z}_{1}^{T} \mathbf{k}^{T} \mathbf{P} \boldsymbol{\Gamma}-\frac{1}{2} \mathbf{z}_{1}^{T} \mathbf{I}_{3 n}+\mathbf{z}_{2}^{T} \boldsymbol{\Gamma}^{T} \mathbf{P} \boldsymbol{\Gamma}\right)\right]\left[\begin{array}{l}
\mathbf{z}_{1} \\
\mathbf{z}_{2}
\end{array}\right] \\
= & \mathbf{z}_{1}^{T} \lambda \mathbf{z}_{1}+\mathbf{z}_{1}^{T} \mathbf{k}^{T} \mathbf{P} \mathbf{k} \mathbf{z}_{1}-\frac{1}{2} \mathbf{z}_{2}^{T} \mathbf{z}_{1} \\
& +\mathbf{z}_{2}^{T} \boldsymbol{\Gamma}^{T} \mathbf{P} \mathbf{k} \mathbf{z}_{1}+\mathbf{z}_{1}^{T} \mathbf{k}^{T} \mathbf{P} \Gamma \mathbf{z}_{2} \\
& -\frac{1}{2} \mathbf{z}_{1}^{T} \mathbf{z}_{2}+\mathbf{z}_{2}^{T} \boldsymbol{\Gamma}^{T} \mathbf{P} \boldsymbol{\Gamma} \mathbf{z}_{2} \\
= & \mathbf{z}_{1}^{T} \lambda \mathbf{z}_{1}-\mathbf{z}_{2}^{T} \mathbf{z}_{1}+\mathbf{s} \mathbf{P s} .
\end{aligned}
$$

Then (36) can be written as

$$
\dot{V}_{2}=-\mathbf{z}^{T} \mathbf{Q} \mathbf{z}-\sum_{r=1}^{3 n} p_{r} \beta_{r}\left|s_{r}\right| \leq 0 .
$$

If we choose the matrix $\gamma$ as small enough, then $\Gamma$ will be positive definite. All the matrices $\lambda, \mathbf{P}$, and $\mathbf{k}$ are diagonal positive definite, if they are chosen to satisfy $\left(\lambda \boldsymbol{\Gamma}^{T}+\mathbf{k}\right) \mathbf{P \Gamma}-$ $(1 / 4) \mathbf{I}_{3 n}>0$; then we can guarantee that the matrix $\mathbf{Q}$ is positive definite. If we define $W(t)=\mathbf{z}^{T} \mathbf{Q z}+\sum_{r=1}^{3 n} p_{r} \beta_{r}\left|s_{r}\right|$, it is obvious that the bounded limit of $W(t)$ exists. From the front definition, we can know that $\mathbf{z}_{1}, \mathbf{z}_{2}$, and $\mathbf{s}$ are bounded. With (16), we can know that $\dot{\mathbf{z}}_{1}$ is bounded. In terms of (22), (26), and (27), $\dot{\mathbf{z}}_{2}$ is bounded. Then $\mathbf{z}$ and $\dot{\mathbf{z}}$ are bounded. Due to the definition of $\mathbf{s}, \mathbf{s}$ and $\dot{s}$ are bounded. Then we can get that $\dot{W}(t)$ is also bounded; then $W(t)$ is uniformly continuous. With Barbalats lemma, $W(t) \rightarrow 0$ as $t \rightarrow \infty$. Then $\mathbf{z}_{1} \rightarrow 0$, $\mathbf{z}_{2} \rightarrow 0, \mathbf{s} \rightarrow 0$ as $t \rightarrow \infty$. With (23), we can get $\varepsilon \rightarrow 0$. Then the synchronization error for each vessel is

$$
\varepsilon_{i}=\sum_{j=1}^{n} a_{i j}\left(\mathbf{z}_{2 j}-\mathbf{z}_{2 i}\right) \longrightarrow 0 .
$$

Because $a_{i j}$ is constant, then we can obtain that $\mathbf{z}_{2 i}(t)-$ $\mathbf{z}_{2 j}(t) \rightarrow 0$.

Though the following calculation

$$
\begin{aligned}
\mathbf{z}_{2 i}(t)-\mathbf{z}_{2 j}(t)= & \dot{\mathbf{z}}_{1 i}+\lambda_{i} \mathbf{z}_{1 i}-\dot{\mathbf{z}}_{1 j}-\lambda_{i} \mathbf{z}_{1 j} \\
= & \left(\dot{\mathbf{x}}_{i}-\dot{\eta}_{d}\right)+\lambda_{i}\left(\mathbf{x}_{i}-\eta_{d}\right) \\
& -\left(\dot{\mathbf{x}}_{j}-\dot{\eta}_{d}\right)-\lambda_{i}\left(\mathbf{x}_{j}-\eta_{d}\right) \\
= & \left(\dot{\mathbf{x}}_{i}-\dot{\mathbf{x}}_{j}\right)+\lambda_{i}\left(\mathbf{x}_{i}-\mathbf{x}_{j}\right) \\
= & \dot{\mathbf{e}}_{i j}+\lambda_{i} \mathbf{e}_{i j},
\end{aligned}
$$

where $\mathbf{e}_{i j}=\mathbf{x}_{i}-\mathbf{x}_{j}$. From the above equation, we can know that it is a linear exponential system with the input as $\mathbf{z}_{2 i}(t)-\mathbf{z}_{2 j}(t)$, and $\mathbf{z}_{2 i}(t)-\mathbf{z}_{2 j}(t) \rightarrow 0$, and $\mathbf{e}_{i j}$ is bounded; then we can obtain that $\lim _{t \rightarrow \infty}\left\|\mathbf{e}_{i j}\right\|=0$; that is $\mathbf{x}_{i}-\mathbf{x}_{j} \rightarrow 0$. According to $\mathbf{z}_{1 i}(t) \rightarrow 0$, then $\mathbf{x}_{i}-\eta_{d} \rightarrow 0$. Finally, we can know that $\mathbf{x}_{1}=\cdots=\mathbf{x}_{i}=\cdots=\mathbf{x}_{n}=\eta_{d}$. So the group of vessels achieve the coordinated tracking while holding the desired formation. 


\section{Robust Formation Controller Design}

In this section, we will design the robust coordinated controller for multiple vessels in the presence of external disturbances. This section will be divided into two parts. The first part is that the upper bounded of the disturbance is known in advance. The second part is that the upper bounded is unknown. For the second part, the adaptive control law is designed to estimate the external disturbances. In this section, for a vector $\mathbf{x} \in \mathrm{R}^{n \times 1}$, the absolute value of the vector is denoted as $|\mathbf{x}|=\left[\left|x_{1}\right|, \ldots,\left|x_{n}\right|\right]^{T}$.

If the external disturbance for each vessel is bounded and the upper bounded satisfy $\left|\tau_{d i}\right| \leq \bar{F}_{i}$, where $\bar{F}_{i} \in \mathrm{R}^{3 \times 1}$ is a positive constant vector, and for multiple vessels, the vector form is $\left|\tau_{d}\right| \leq \bar{F}$. If we choose the control input as

$$
\tau=\tau_{\mathrm{eq}}+\tau_{\mathrm{sw}}+\tau_{\mathrm{en}},
$$

where $\tau_{\mathrm{eq}}$ and $\tau_{\mathrm{sw}}$ are the same with the definition in the front section and $\tau_{\text {en }}$ is the control input to compensate for the external disturbances, the detailed representation is

$$
\tau_{\text {en }}=-\left[\begin{array}{llll}
\bar{F}_{1} \operatorname{sign}\left(s_{1}\right) & \cdots & \bar{F}_{3 n} \operatorname{sign}\left(s_{3 n}\right)
\end{array}\right]^{T}=-\mathbf{F} .
$$

For each vessel, the control input to compensate the external disturbance is as follows

$$
\tau_{\text {en }}^{i}=-\mathbf{F}_{i} \in \mathrm{R}^{3 \times 1} .
$$

Theorem 5. Consider $n$ vessels to perform the coordination formation task, the vessel model is described as (3) and (4). with the distributed coordinated formation control law (42), (27), and (43), and the conditions in Theorem 4 are satisfied, then the position tracking error, the velocity tracking error and their synchronization error, are asymptotically stable; that is, these vessels can realize the coordination formation asymptotically.

Proof. The proof procedure in this section is similar to the front section. The main difference is that the external disturbances are considered in this section.

Define the same Lyapunov function with the front section as

$$
V_{2}=V_{1}+\frac{1}{2} \mathbf{s}^{T} \mathbf{s}
$$

Taking the time derivative of the above equation as

$$
\begin{aligned}
\dot{V}_{2}= & \dot{V}_{1}+\mathbf{s}^{T} \dot{\mathbf{s}} \\
= & \dot{V}_{1}+\mathbf{s}^{T} \mathbf{k} \dot{\mathbf{z}}_{1}+\mathbf{s}^{T}\left(\mathbf{I}_{3 n}+\gamma\left(\mathbf{L} \otimes \mathrm{I}_{3}\right)\right) \dot{\mathbf{z}}_{2} \\
= & \mathbf{z}_{1}^{T} \mathbf{z}_{2}-\mathbf{z}_{1}^{T} \lambda \mathbf{z}_{1}+\mathbf{s}^{T} \mathbf{k}\left(\mathbf{z}_{2}-\lambda \mathbf{z}_{1}\right) \\
& +\mathbf{s}^{T}\left(\mathbf{I}_{3 n}+\gamma\left(\mathbf{L} \otimes \mathrm{I}_{3}\right)\right) \mathbf{M}_{n}^{-1}(\eta) \\
& \times\left[\tau+\tau_{d}-\mathbf{M}_{n}(\eta) \ddot{\eta}_{D}+\mathbf{M}_{n}(\eta) \dot{\mathbf{f}}+\mathbf{M}_{n}(\eta) \lambda \dot{\mathbf{z}}_{1}\right. \\
& \quad-\left(\mathbf{C}_{n}(\eta, \dot{\eta})+\mathbf{D}_{n}(\eta, \dot{\eta})\right)\left(\dot{\eta}_{D}-\dot{\mathbf{f}}\right) \\
& \left.\quad-\left(\mathbf{C}_{n}(\eta, \dot{\eta})+\mathbf{D}_{n}(\eta, \dot{\eta})\right)\left(\mathbf{z}_{2}-\lambda \mathbf{z}_{1}\right)\right] ;
\end{aligned}
$$

with the control input (42), (27), and (43) yield:

$$
\begin{aligned}
\dot{V}_{2}= & -\mathbf{z}_{1}^{T} \lambda \mathbf{z}_{1}+\mathbf{z}_{1}^{T} \mathbf{z}_{2}-\mathbf{s}^{T} \mathbf{P}(\mathbf{s}+\beta \operatorname{sign}(s)) \\
& +\mathbf{s}^{T}\left(\mathbf{I}_{3 n}+\gamma\left(\mathbf{L} \otimes \mathrm{I}_{3}\right)\right) \mathbf{M}_{n}^{-1}(\eta)\left(\tau_{d}-\mathbf{F}\right) \\
\leq & -\mathbf{z}_{1}^{T} \lambda \mathbf{z}_{1}+\mathbf{z}_{1}^{T} \mathbf{z}_{2}-\mathbf{s}^{T} \mathbf{P} \mathbf{s}-\sum_{r=1}^{3 n} p_{r} \beta_{r}\left|s_{r}\right| \\
& +|\mathbf{s}|\left(\mathbf{I}_{3 n}+\gamma\left(\mathbf{L} \otimes \mathrm{I}_{3}\right)\right) \mathbf{M}_{n}^{-1}(\eta)\left(\left|\tau_{d}\right|-\overline{\mathbf{F}}\right) \\
\leq & -\mathbf{z}_{1}^{T} \lambda \mathbf{z}_{1}+\mathbf{z}_{1}^{T} \mathbf{z}_{2}-\mathbf{s}^{T} \mathbf{P s}-\sum_{r=1}^{3 n} p_{r} \beta_{r}\left|s_{r}\right| .
\end{aligned}
$$

From the above inequality, we can see that the same results with the front section are achieved; then we can prove that the position tracking error, velocity tracking error, and their synchronization error are asymptotically stable according to the proof procedure in the front section.

If the external disturbances are unknown in advance, and we assume that the external disturbances vary slowly; that is $\dot{\tau}_{d}=0$, then we adopt the adaptive control to estimate the disturbances. Then control law also includes three parts, which is written as

$$
\tau=\tau_{\mathrm{eq}}+\tau_{\mathrm{sw}}+\tau_{\mathrm{en}}
$$

where

$$
\tau_{\text {en }}=-\widehat{\tau}_{d},
$$

and $\widehat{\tau}_{d}$ is the estimate value of the external disturbances. The adaptive control law is chosen as follows

$$
\dot{\hat{\tau}}_{d}=\sigma \mathbf{M}_{n}\left(\mathbf{I}_{3 n}+\gamma\left(\mathbf{L} \otimes \mathrm{I}_{3}\right)\right)^{-1} \mathbf{s},
$$

where $\sigma$ is a positive real number.

Theorem 6. Consider a group of $n$ vessels to perform the coordination formation task, the vessel model is described as (3) and (4). With the coordinated formation control law (42), (27), and (49), and the adaptive control law (50) and the conditions in the Theorem 4 are satisfied, then the position tracking error, the velocity tracking error, and their synchronization error are asymptotically stable; that is these vessels can realize the coordination formation asymptotically.

Proof. Define the new Lyapunov function as

$$
V_{3}=V_{2}+\frac{1}{2 \sigma} \widetilde{\tau}_{d}^{T} \widetilde{\tau}_{d}
$$

where the definition of $V_{2}$ is same as the front one, and $\tilde{\tau}_{d}$ is defined as the estimated error, the form of which is $\widetilde{\tau}_{d}=$ $\tau_{d}-\widehat{\tau}_{d}$, where $\widehat{\tau}_{d}$ is the estimated value of the disturbances.

Take the time derivative of the previous equation as

$$
\begin{aligned}
\dot{V}_{3}= & \dot{V}_{1}+\mathbf{s}^{T} \dot{\mathbf{s}}+\frac{1}{\sigma} \tilde{\tau}_{d}^{T} \dot{\widetilde{\tau}}_{d} \\
= & \mathbf{z}_{1}^{T} \mathbf{z}_{2}-\mathbf{z}_{1}^{T} \lambda \mathbf{z}_{1}+\mathbf{s}^{T} \mathbf{k} \dot{\mathbf{z}}_{1} \\
& +\mathbf{s}^{T}\left(\mathbf{I}_{3 n}+\gamma\left(\mathbf{L} \otimes \mathrm{I}_{3}\right)\right) \dot{\mathbf{z}}_{2}-\frac{1}{\sigma} \tilde{\tau}_{d}^{T} \dot{\vec{\tau}}_{d}
\end{aligned}
$$




$$
\begin{aligned}
= & \mathbf{z}_{1}^{T} \mathbf{z}_{2}-\mathbf{z}_{1}^{T} \lambda \mathbf{z}_{1}+\mathbf{s}^{T} \mathbf{k}\left(\mathbf{z}_{2}-\lambda \mathbf{z}_{1}\right) \\
& +\mathbf{s}^{T}\left(\mathbf{I}_{3 n}+\gamma\left(\mathbf{L} \otimes \mathrm{I}_{3}\right)\right) \mathbf{M}_{n}^{-1}(\eta) \\
& \times\left[\tau+\tau_{d}-\mathbf{M}_{n}(\eta) \ddot{\eta}_{d}+\mathbf{M}_{n}(\eta) \dot{\mathbf{f}}+\mathbf{M}_{n}(\eta) \lambda \dot{\mathbf{z}}_{1}\right. \\
& -\left(\mathbf{C}_{n}(\eta, \dot{\eta})+\mathbf{D}_{n}(\eta, \dot{\eta})\right)\left(\dot{\eta}_{d}-\dot{\mathbf{f}}\right) \\
& \left.-\left(\mathbf{C}_{n}(\eta, \dot{\eta})+\mathbf{D}_{n}(\eta, \dot{\eta})\right)\left(\mathbf{z}_{2}-\lambda \mathbf{z}_{1}\right)\right] \\
& -\frac{1}{\sigma} \widetilde{\tau}_{d}^{T} \dot{\hat{\tau}}_{d} \\
= & -\mathbf{z}_{1}^{T} \lambda \mathbf{z}_{1}+\mathbf{z}_{1}^{T} \mathbf{z}_{2}-\mathbf{s}^{T} \mathbf{P}(\mathbf{s}+\beta \operatorname{sign}(\mathbf{s})) \\
& -\frac{1}{\sigma} \widetilde{\tau}_{d}^{T}\left(\dot{\hat{\tau}}_{d}-\sigma \mathbf{M}_{n}(\eta)\left(\mathbf{I}_{3 n}+\gamma\left(\mathbf{L} \otimes \mathrm{I}_{3}\right)\right)^{-1} \mathbf{s}\right) \\
= & -\mathbf{z}_{1}^{T} \lambda \mathbf{z}_{1}+\mathbf{z}_{1}^{T} \mathbf{z}_{2}-\mathbf{s}^{T} \mathbf{P}(\mathbf{s}+\beta \operatorname{sign}(\mathbf{s})) \\
= & -\mathbf{z}_{1}^{T} \lambda \mathbf{z}_{1}+\mathbf{z}_{1}^{T} \mathbf{z}_{2}-\mathbf{s}^{T} \mathbf{P s}-\sum_{r=1}^{3 n} p_{r} \beta_{r}\left|s_{r}\right| .
\end{aligned}
$$

From the above equality, we can see that the same results with the front section are achieved; then we can prove that the position tracking error, velocity tracking error and their synchronization error, are asymptotically stable according to the proof procedure in the front section.

\section{Simulation Results}

In this section, experimental simulations are carried out to evaluate the effectiveness of the proposed coordinated formation control algorithm. Five marine vessels are considered to perform the coordinated tracking task. Detailed parameters of these vessels are presented in [12]. The proposed algorithm has achieved the leaderless coordination based on the graph theory. In this experiment, the topology graph of communication among these five vessels is chosen as Figure 1.

And the Laplacian matrix of the communication topology graph is as follows

$$
L=\left[\begin{array}{ccccc}
1 & 0 & 0 & 0 & -1 \\
-1 & 3 & 0 & -1 & -1 \\
0 & -1 & 1 & 0 & 0 \\
0 & 0 & -1 & 1 & 0 \\
0 & 0 & 0 & -1 & 1
\end{array}\right]
$$

The initial positions of the five vessels are $\eta_{1}=[-80$ $800-7 \pi / 30]^{T}, \eta_{2}=\left[\begin{array}{lll}-30 & 800 & -\pi / 3\end{array}\right]^{T}, \eta_{3}=\left[\begin{array}{ll}-100 & 780\end{array}\right.$ $-\pi / 4]^{T}, \eta_{4}=\left[\begin{array}{lll}20 & 830 & -\pi / 2\end{array}\right]^{T}$, and $\eta_{5}=\left[\begin{array}{lll}-100 & 800\end{array}\right.$ $-\pi / 4]^{T}$, respectively. In order to evaluate the performance of the coordinated tracking, the desired formation pattern of the coordinated formation controller is described by $\mathbf{l}_{1}=$ $\left[\begin{array}{lll}0 & 0 & 0\end{array}\right]^{T}, \mathbf{l}_{2}=\left[\begin{array}{lll}0 & 50 & 0\end{array}\right]^{T}, \mathbf{l}_{3}=\left[\begin{array}{lll}0 & -50 & 0\end{array}\right]^{T}, \mathbf{l}_{4}=\left[\begin{array}{lll}0 & 100 & 0\end{array}\right]^{T}$, and $\mathbf{1}_{5}=\left[\begin{array}{lll}0 & -100 & 0\end{array}\right]^{T}$. The desired trajectory for all the formation points is chosen as $\eta_{d}(t)=\left[\begin{array}{lll}n_{d} & e_{d} & \psi_{d}\end{array}\right]^{T}$, and the detailed forms are $n_{d}=t, e_{d}=800-800 \sin (t / 400)$, and $\psi_{d}=\arctan \left(\dot{e}_{d} / \dot{n}_{d}\right)$.

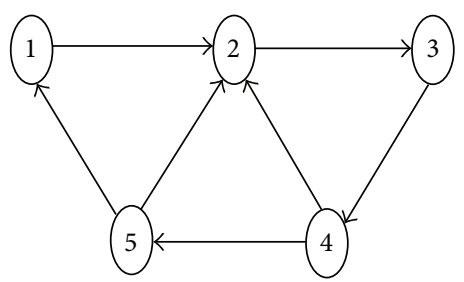

FIGURE 1: The communication topology graph of these vessels.

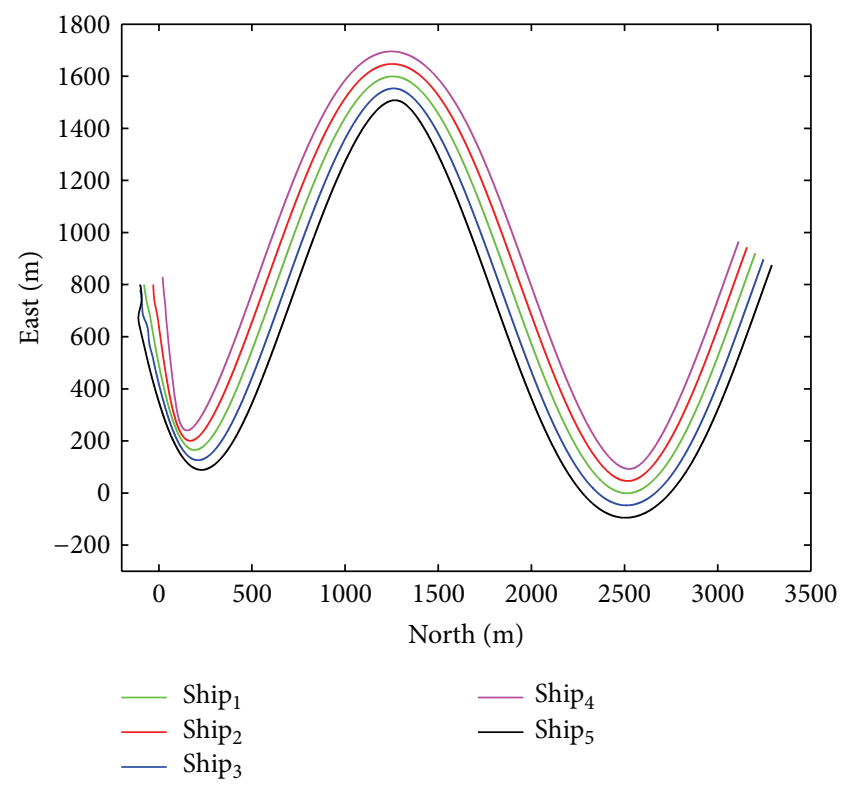

FIGURE 2: The formation trajectories in horizontal plane.

Although the proposed coordination algorithm is robust to unknown external disturbance, the disturbance model in the simulation can be chosen as a fixed term instead of the unknown disturbance. We assume that the vessels encounter the wind, wave, and current. And the wind is assumed to be fixed direction and fixed velocity; then the disturbance of wind is a constant; the wave and current is assumed to be the sine wave with a fixed frequency at one time. So the external disturbances can be chosen as

$$
\begin{gathered}
\tau_{d i}=10^{6} *\left[0.05 \sin \left(\frac{\pi t}{100}\right)+0.02,0.03 \sin \left(\frac{\pi t}{100}\right),\right. \\
\left.0.01 \sin \left(\frac{\pi t}{100}\right)+0.01\right]^{T}(N) .
\end{gathered}
$$

The control parameters of the coordinated formation controller are chosen as $\lambda_{i}=\operatorname{diag}(0.05,0.05,0.2)$, $\gamma_{i}=\operatorname{diag}(1,1,1), \mathbf{P}_{i}=\operatorname{diag}(0.3,0.3,0.15)$, and $\beta_{i}=$ $\operatorname{diag}(1,1,0.5)$.

The simulation results are shown from Figure 2 to Figure 9. Figure 2 shows the movements for these vessels in the plane. The heading change curve of each vessel is shown in Figure 3. Figures 4, 5, and 6 show the surge velocity, the sway velocity, and the angular velocity of each vessel during the coordinated control process, respectively. Figures 7, 8, and 9 


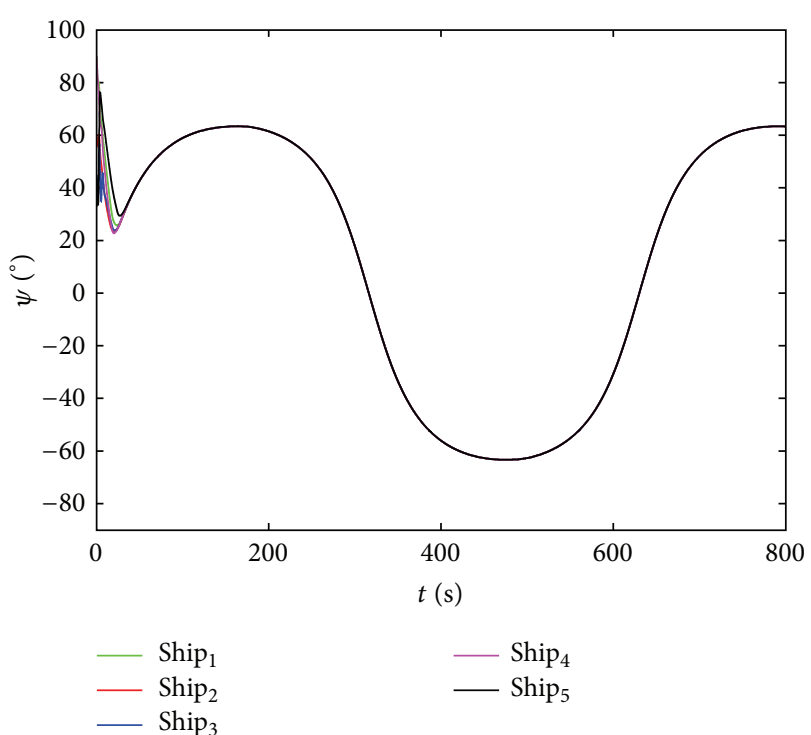

FIGURE 3: The heading curve of each vessel.

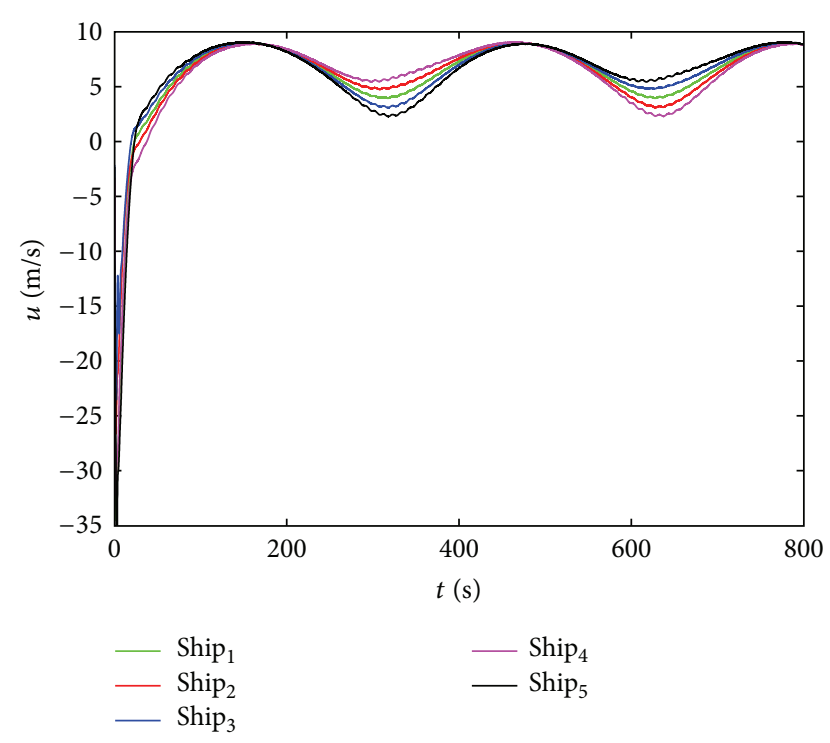

FIGURE 4: The surge velocity curve of each vessel.

show the surge force, sway forces, and the heave torques applied to the vessels, respectively.

We can see that these vessels realized the coordinated tracking task from Figures 2 and 3. From Figures 4, 5, and 6 , the velocities of these vessels achieve consensus as a whole, and the velocities cannot achieve consensus when the vessels move to the inflexion of the respective path curve. The phenomenon of the surge velocity is obvious in the experimental simulations due to the vessels move along the tangent directions of the desired trajectory. This phenomenon appears as a result of vessel speed regulated to maintain the desired formation pattern at the inflexion of the respective path curve. From Figures 7, 8, and 9, we know that the forces and torques for the group of $n$ vessels approach consensus, in a way. This is the result of that these vessels are uniform in

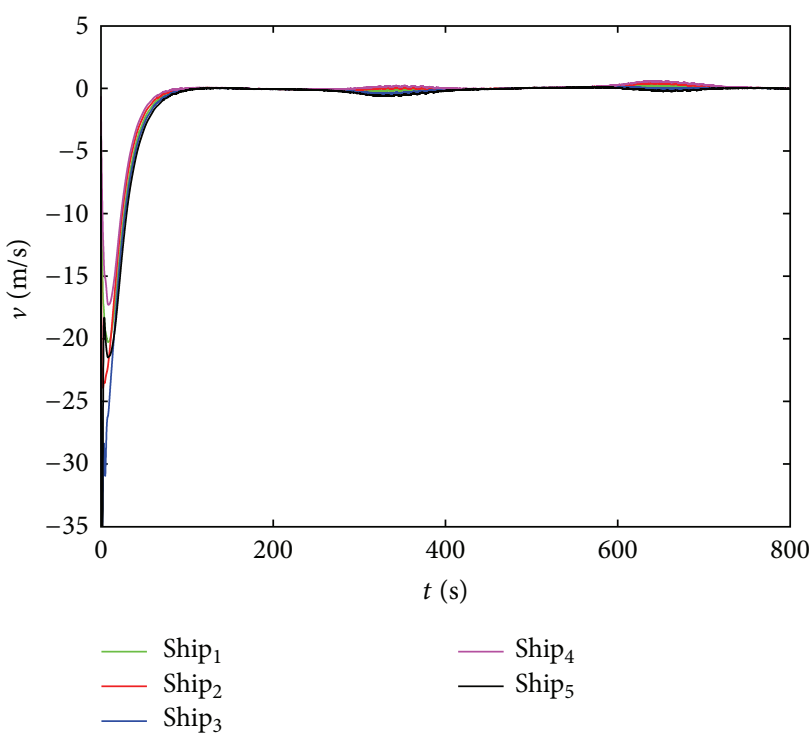

Figure 5: The sway velocity curve of each vessel.

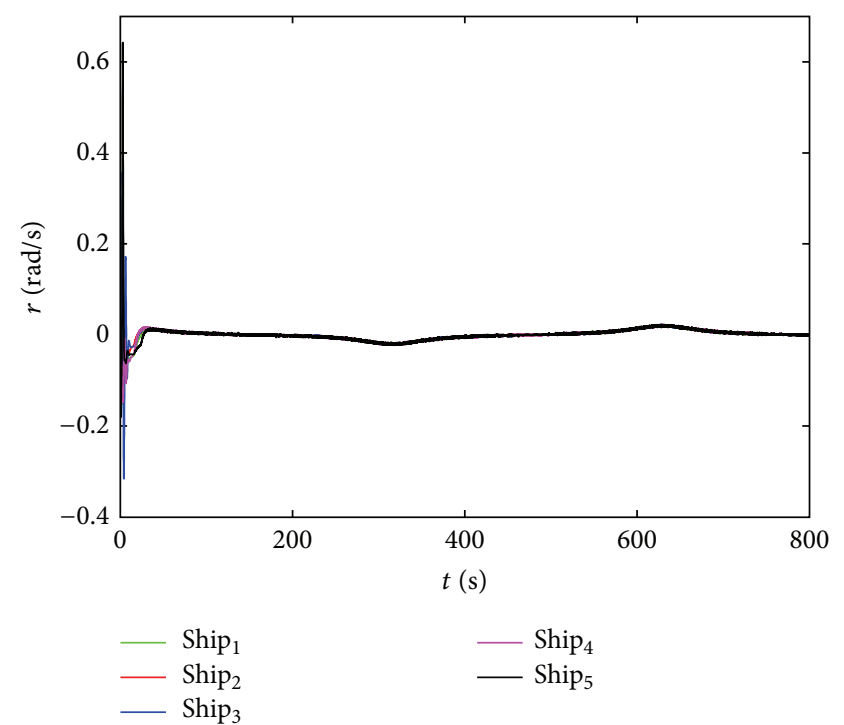

FIGURE 6: The heave velocity curve of each vessel.

these experimental simulations. With the analysis of the simulation results, we can conclude that these vessels can accomplish coordinated trajectory tracking task while keeping the desired formation. It means that the proposed coordination control algorithm is effective.

\section{Conclusion}

This paper has proposed a new backstepping sliding mode coordinated formation control algorithm for multiple surface vessels in the presence of external environmental disturbances. The proposed coordinated formation controller for these vessels is designed by defining a new switched function. And a new cross-coupling error is defined using the velocity error and velocity synchronization error to be applicable 


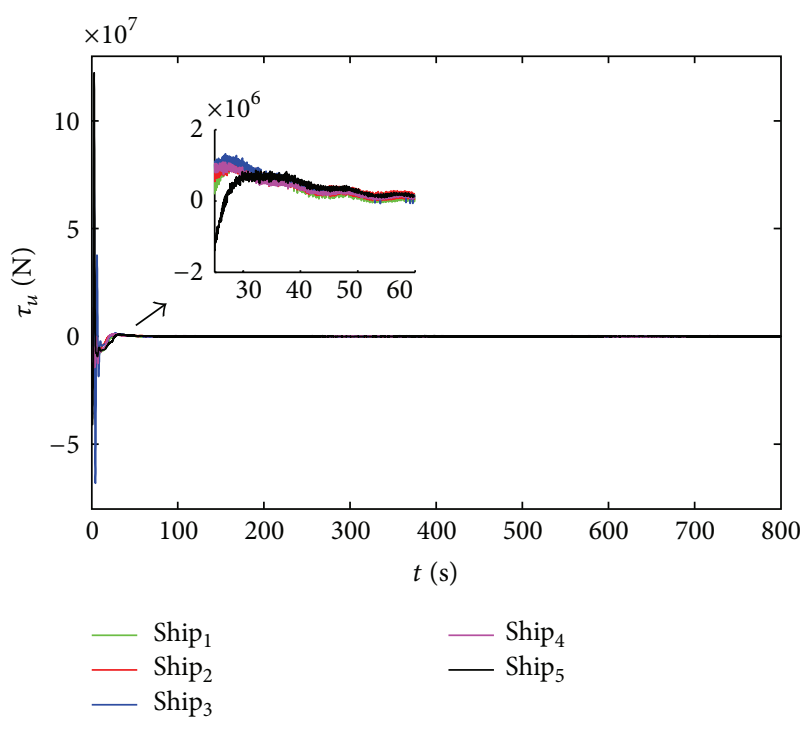

FIGURE 7: The surge force of each vessel.

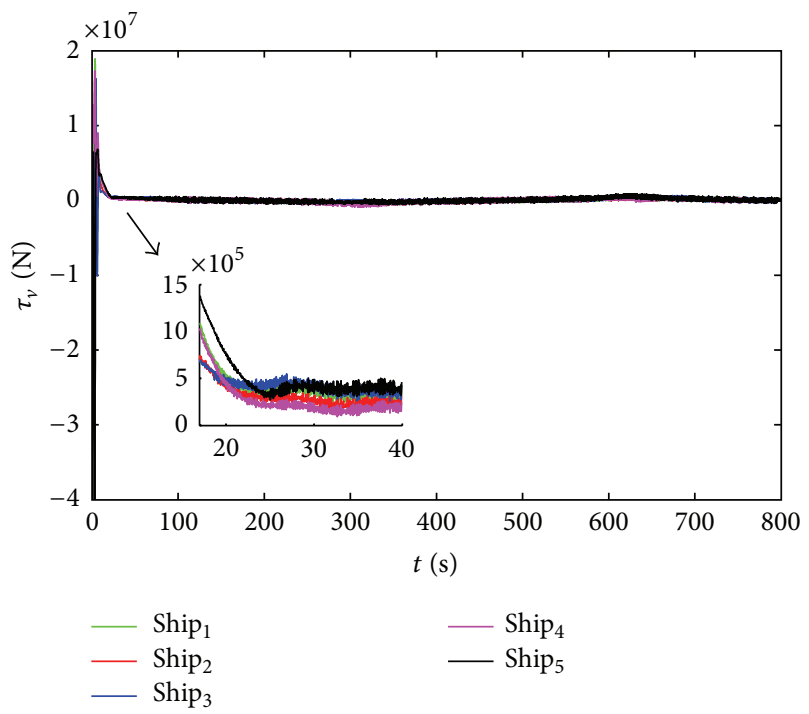

Figure 8: The sway force of each vessel.

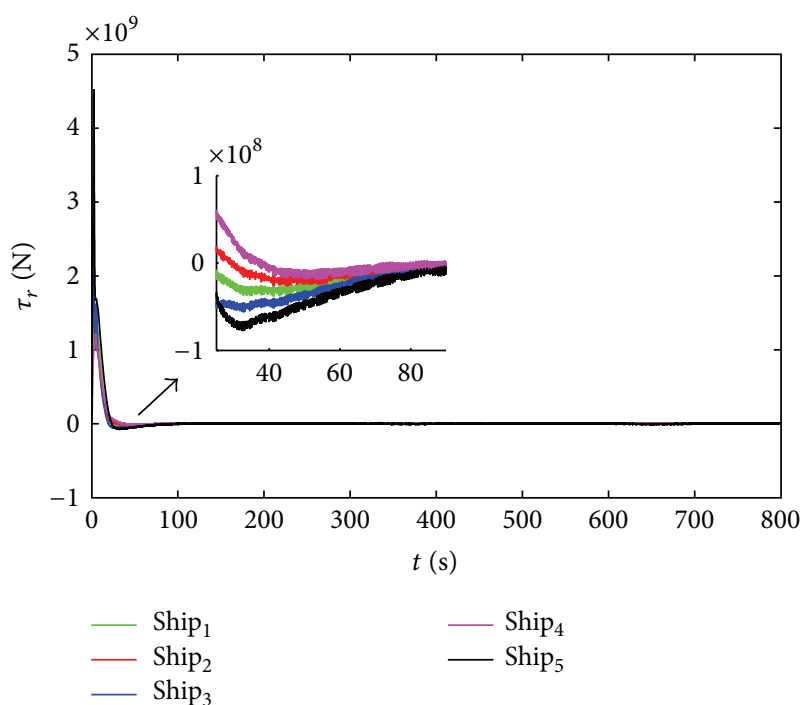

FIgURE 9: The heave force of each vessel. for the backstepping sliding mode controller. In addition, the adaptive control law is also designed to compensate for the external disturbances and then achieve the robustness. Finally, the effectiveness of the proposed coordination control algorithm is demonstrated by experimental simulations.

\section{References}

[1] L. Zhang, H. Gao, and O. Kaynak, "Network-induced constraints in networked control system-a survey," IEEE Transactions on Industrial Informatics, vol. 9, no. 1, pp. 403-416, 2013.

[2] W. Ren, R. W. Beard, and E. M. Atkins, "A survey of consensus problems in multi-agent coordination," in Proceedings of the American Control Conference (ACC '05), pp. 1859-1864, June 2005.

[3] A. Aguiar, J. Almeida, and M. Bayat, "Cooperative autonomous marine vehicle motion control in the scope of the eugrex project: theory and practice," in Proceedings of the IEEE/MTS Conference on Oceans, pp. 1-10, 2009.

[4] I.-A. F. Ihle, J. Jouffroy, and T. I. Fossen, "Formation control of marine surface craft: a lagrangian approach," IEEE Journal of Oceanic Engineering, vol. 31, no. 4, pp. 922-934, 2006.

[5] F. Arrichiello, S. Chiaverini, and T. I. Fossen, "Formation control of underactuated surface vessels using the null-spaee-based behavioral control," in Proceedings of the IEEE/RSJ International Conference on Intelligent Robots and Systems (IROS '06), pp. 5942-5947, October 2006.

[6] F. Fahimi, "Non-linear model predictive formation control for groups of autonomous surface vessels," International Journal of Control, vol. 80, no. 8, pp. 1248-1259, 2007.

[7] J. Almeida, C. Silvestre, and A. M. Pascoal, "Cooperative control of multiple surface vessels with discrete-time periodic communications," International Journal of Robust and Nonlinear Control, vol. 22, no. 4, pp. 398-419, 2012.

[8] I.-A. F. Ihle, M. Arcak, and T. I. Fossen, "Passivity-based designs for synchronized path-following," Automatica, vol. 43, no. 9, pp. 1508-1518, 2007.

[9] E. Børhaug, A. Pavlov, E. Panteley, and K. Y. Pettersen, "Straight line path following for formations of underactuated marine surface vessels," IEEE Transactions on Control Systems Technology, vol. 19, no. 3, pp. 493-506, 2011.

[10] J. Ghommam and F. Mnif, "Coordinated path-following control for a group of underactuated surface vessels," IEEE Transactions on Industrial Electronics, vol. 56, no. 10, pp. 3951-3963, 2009.

[11] Y. Wang, W. Yan, and J. Li, "Passivity-based formation control of autonomous underwater vehicles," IET Control Theory \& Applications, vol. 6, no. 4, pp. 518-525, 2012.

[12] C. Thorvaldsen and R. Skjetne, "Formation control of fullyactuated marine vessels using group agreement protocols," in Proceedings of the 50th IEEE Conference on Decision and Control and European Control Conference, pp. 4132-4139, 2011.

[13] M. Fu and J. Jiao, "A hybrid approach for coordinated formation control of multiple surface vessels," Mathematical Problems in Engineering, vol. 2013, Article ID 794284, 8 pages, 2013.

[14] I. A. Gravagne, J. M. Davis, and J. J. DaCunha, "A unified approach to high-gain adaptive controllers," Abstract and Applied Analysis, vol. 2009, Article ID 198353, 13 pages, 2009.

[15] S. Yin, S. Ding, and H. Luo, "Real-time implementtion of fault tolerant control system with performance optimization," IEEE Transactions on Industrial Electronics, 2013. 
[16] J. Huang, H. Li, Y. Chen, and Q. Xu, "Robust position control of PMSM using fractional-order sliding mode controller," Abstract and Applied Analysis, vol. 2012, Article ID 512703, 33 pages, 2012.

[17] L. Zhang and E.-K. Boukas, "Stability and stabilization of Markovian jump linear systems with partly unknown transition probabilities," Automatica, vol. 45, no. 2, pp. 463-468, 2009.

[18] L. Zhang, P. Shi, E.-K. Boukas, and C. Wang, " $\mathrm{H}_{\infty}$ model reduction for uncertain switched linear discrete-time systems," Automatica, vol. 44, no. 11, pp. 2944-2949, 2008.

[19] E. A. Tannuri, A. C. Agostinho, H. M. Morishita, and L. Moratelli, "Dynamic positioning systems: an experimental analysis of sliding mode control," Control Engineering Practice, vol. 18, no. 10, pp. 1121-1132, 2010.

[20] H. Ashrafiuon, K. R. Muske, L. C. McNinch, and R. A. Soltan, "Sliding-mode tracking control of surface vessels," IEEE Transactions on Industrial Electronics, vol. 55, no. 11, pp. 4004-4012, 2008.

[21] F. Fahimi, "Sliding-mode formation control for underactuated surface vessels," IEEE Transactions on Robotics, vol. 23, no. 3, pp. 617-622, 2007.

[22] M. Defoort, T. Floquet, A. Kökösy, and W. Perruquetti, "Slidingmode formation control for cooperative autonomous mobile robots," IEEE Transactions on Industrial Electronics, vol. 55, no. 11, pp. 3944-3953, 2008.

[23] Y. Xia, Z. Zhu, and M. Fu, "Back-stepping sliding mode control for missile systems based on an extended state observer," IET Control Theory \& Applications, vol. 5, no. 1, pp. 93-102, 2011.

[24] D. Zhao and T. Zou, "A finite-time approach to formation control of multiple mobile robots with terminal sliding mode," International Journal of Systems Science, vol. 43, no. 11, pp. 19982014, 2012.

[25] D. Zhao, T. Zou, S. Li, and Q. Zhu, "Adaptive backstepping sliding mode control for leader-follower multi-agent systems," IET Control Theory \& Applications, vol. 6, no. 8, pp. 1109-1117, 2012.

[26] W. Ren, "Distributed leaderless consensus algorithms for networked Euler-Lagrange systems," International Journal of Control, vol. 82, no. 11, pp. 2137-2149, 2009.

[27] D. Sun, C. Wang, W. Shang, and G. Feng, "A synchronization approach to trajectory tracking of multiple mobile robots while maintaining time-varying formations," IEEE Transactions on Robotics, vol. 25, no. 5, pp. 1074-1086, 2009.

[28] H. Khalil, Nonlinear Systems, Prentice Hall, Upper Saddle River, NJ, USA, 3rd edition, 2002.

[29] T. Fossen, Marine Control Systems: Guidance, Navigation and Control of Ships, Rigs and Underwater Vehicles, Marine Cybernetics, Trondheim, Norway, 2002. 


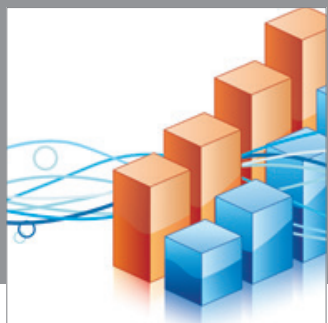

Advances in

Operations Research

mansans

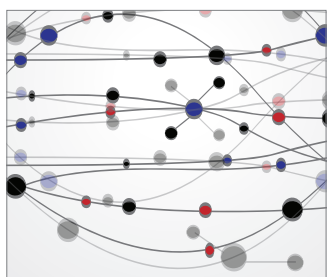

The Scientific World Journal
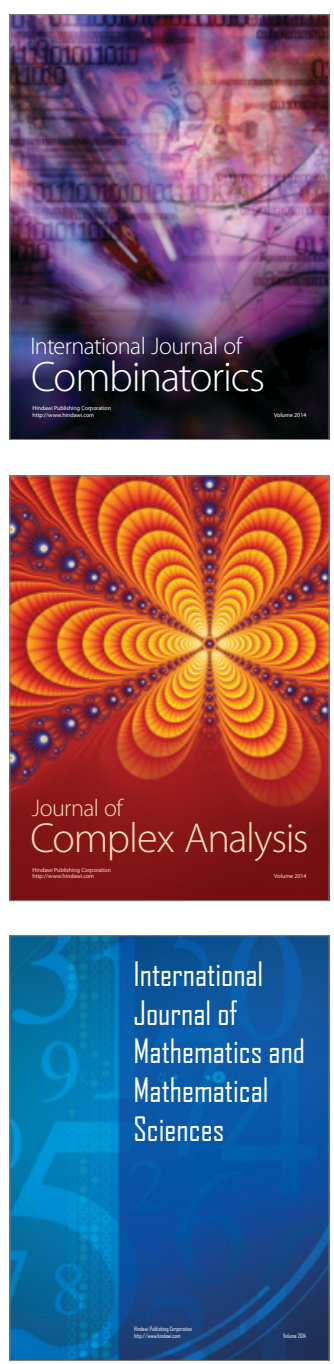
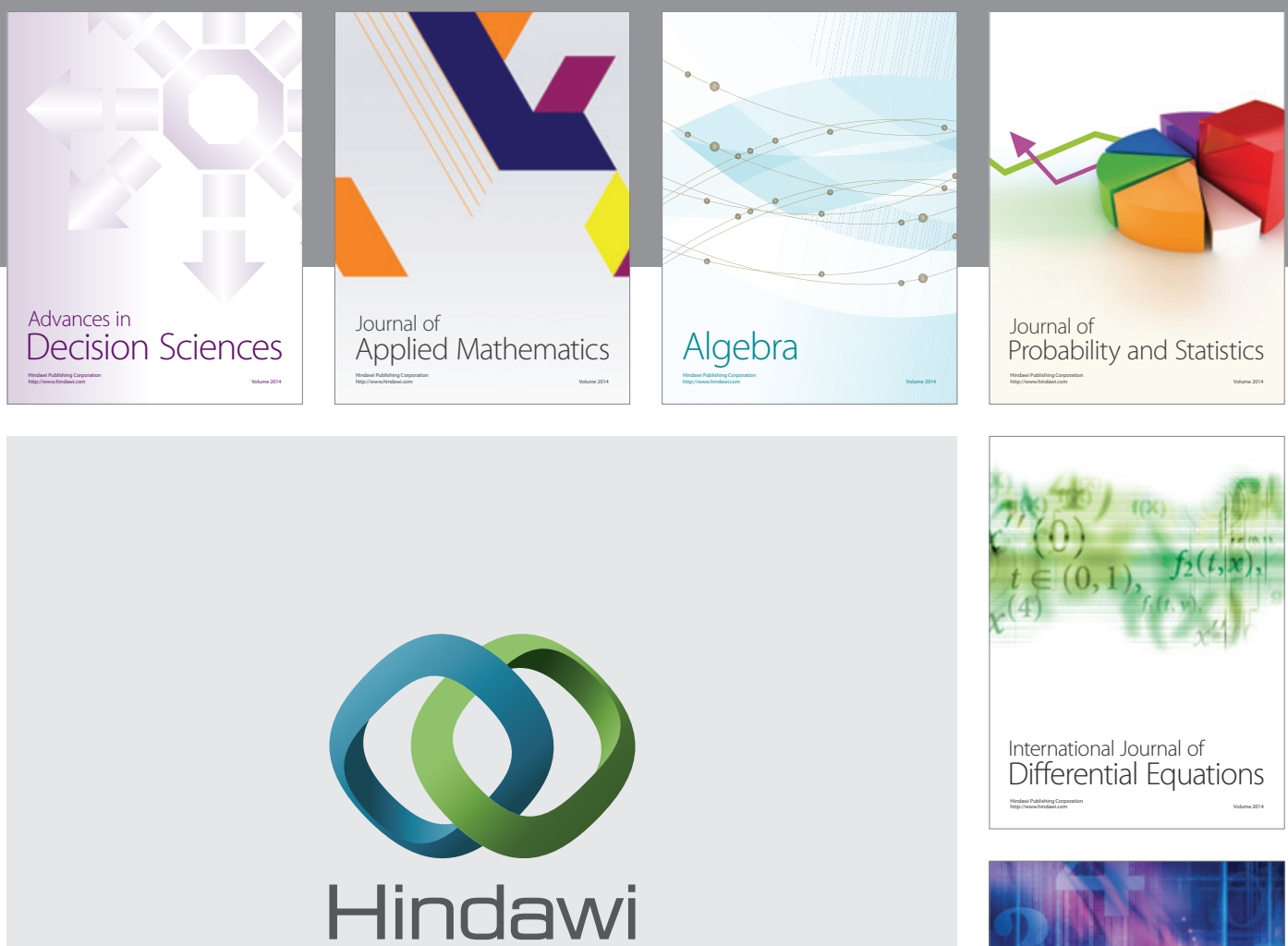

Submit your manuscripts at http://www.hindawi.com
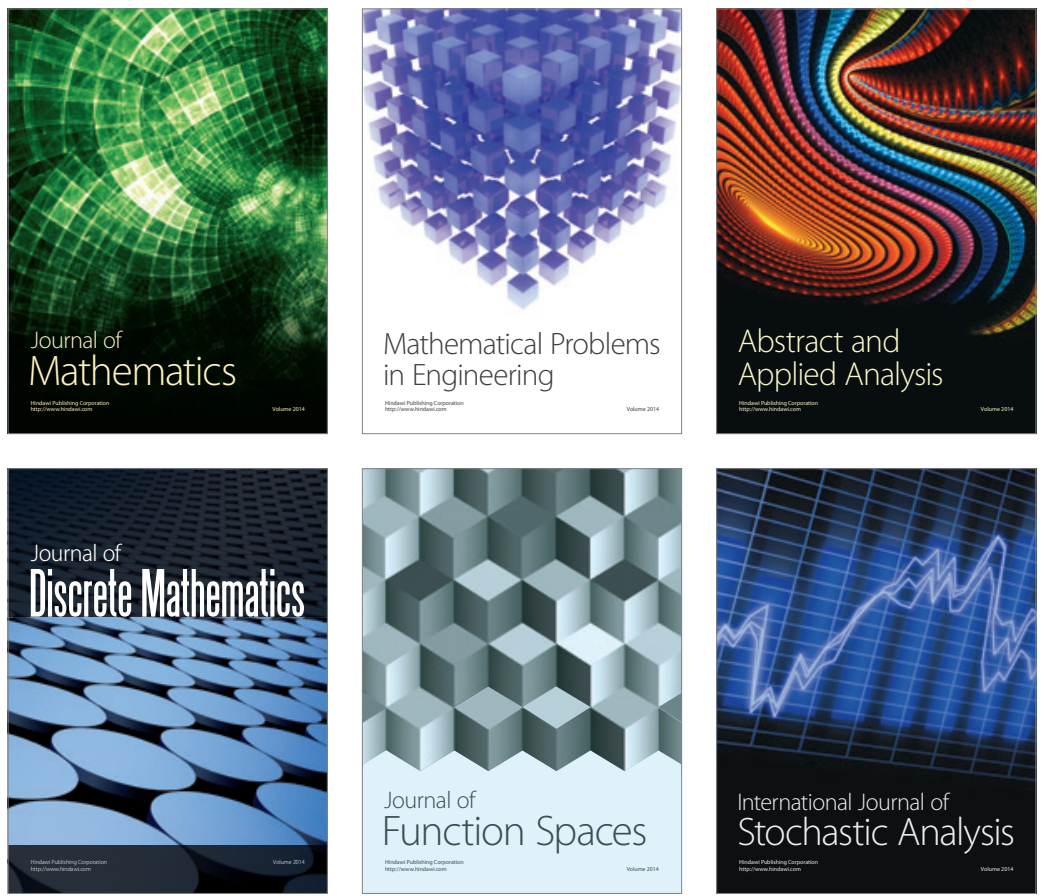

Journal of

Function Spaces

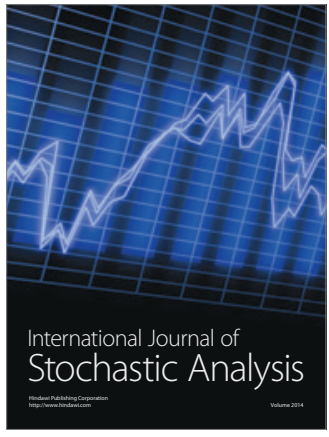

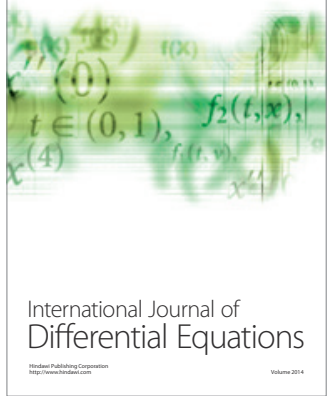
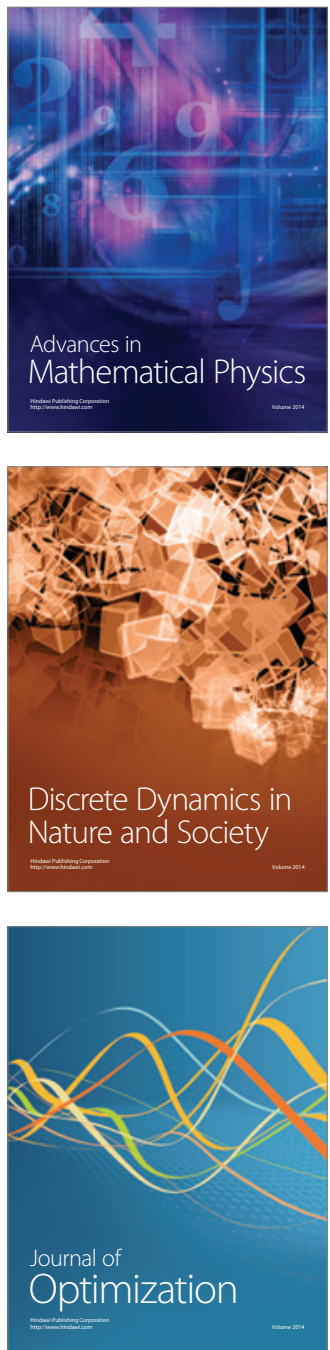